Pour éviter le glissement des remblais dans la fouille, on a exxécuté de distance en distance des murs en maçonnerie reposant sur les massifs de fondation dos sommiers, lorsque le terain ćtait particulièrement déclive.

Aux deux extrémités de chaque siphon, la conduite générale débouche dans un petit bassin qui est logé dans une maisonnette, dont une est visible sur la photographie ci-jointe. Un systeme de deux vannes, correspondant chacune à un tuyau, permet d'interrompre à volonté l'admission de l'eau dans l'une ou l'autre des canalisations ou dans toutes les deux à la fois.

Tolles sont les principales données de cette grande et belle entreprise, comme on le voit intéressante au premier chef à suivre dans los détails techniques de son exécution. Nous y reviendrons lorsque les documents dont nous avons parlé au début de celte notice seront divulguis.

E.-F. CÓTE.

\section{INDICATEUR D'ÉTAT DE CHARGE d'un conducteur électrique ${ }^{(*)}$}

Des ouvriers électriciens, des contremaîtres, des ingénieurs même, sont chaque année victimes d'accidents, au cours de leurs travaux, par suite de l'ignorance dans laquelle ils se sont trouvés de l'état de charge de conducteurs d'énergie électrique au moment où il était nécessaire d'y faire un travail quelconque et de l'impossibilité de constater rapidement cet état lui-même.

Le Ministre du commerce, de l'industrie, des postes et des télégraphes, justement préoccupé de la gravité de ces accidents, avait, d'accord avec le Comité d'électricité, prié un certain nombre de Sociétés ou d'associations s'intéressant aux applications de l'électricité, de mettre à l'étude un appareil portatif permettant de reconnat̂tre, par une simple observation, l'étal de charge d'un conducteurélectrique.

L'Association des Industriels de France contre les accidents du travail avait été désignée par ces différents groupements, pour organiser, dans ce but, un concours public international et pour réunir les fonds nécessaires.

Une Commission spéciale du concours avait été constituée et elle avait dressé le programme suivant:

Programme. - L'appareil faisant l'objet du concours est destiné à être mis entre les mains de toutes personnes ayant à effectuer des travaux sur des canalisations électriques ou à promixité de celles-ci, de façon à leur permettre de s'assurer d'une manière permanente qu'elles ne courront aucun danger en touchant à ces canalisations.

L'appareil doit être robuste, d'un transport et d'un maniemenc faciles; son fonctionnement ne doit pas être troublé par les agents almosphérique et ses indications doivent toujours être très sûres en tout temp̧s et en toute circonstance.

Si l'appareil est mis directement ou indirectement en contact avec un ou plusieurs conducteurs chargés, il ne doit pouroir en résulter aucun accident pour l'operateur, l'appareil ou le réseau de distribution. Il ne doit survenir aucune gêne dans le réseau de distribution de la mise en contact de l'appareil avec une canalisation, ni de son fonctionnement.

Le même appareıl doit également s'appliquer aux distributions à courant continu et courants alternatifs à basse tension et à haute tension, aux canalisations aériennes et souterraines.

$\left({ }^{*}\right)$ D'après le rapport-présenté par $\mathrm{M}$. Charles Roux à l'CAssociation des Industriels de France contre les accidents du trayal.
Grâce au concours de généreux donateurs, une somme de 6ooo fr . avait été affectée aux prix à décerner aux appareils reconnus les meilleurs.

Dix-huit concurrents avaient répondu à l'appel qui leur était adressé.

Après une première étude des dispositifs présentés au concours, la Commission d'examen en a retenu ıo, présen. tés par 8 concurrents, pour être soumis aux épreuves pratiques. A la suite de ces épreuves les conclusions de la Commission ont été les suivantes:

Pas de premier prix.

Un deuxième prix, de 3.500 francs, a M. Maurice Miet, ancien ingénieur du secteur de la Rive gauche, à Paris.

Un troisième prix, de r.ooo francs, à M. J.-B. Taylor, de Schenecrady (Etats-Unis).

Deux mentions honorables, de 500 francs, à $M$. William Thornton, de Newcastle, et à la Minerallac Company de Chicago.

A ppareil de M. Miet. - Cet appareil se compose (fig. I) d'un tube de verre épais $A$ fermé à une extrémité (éprouvette de laboratoire).

Ce tube porte un bouchon en caoutchouc B laissant passer une tige de laiton $C$ terminée par une boule de cuivre $D$ à l'extérieur et par deux minces feuilles d'aluminium $\mathrm{E}$ à la partie inférieure.

En tenant l'éprouvette à la main par la partie $F$ et approchant la boule D d'un conducteur en charge, les feulles d'aluminium s'écartent comme le montre la figure.

L'écart est d'autant plus grand que la tension de ligne essayée est plus élevée. C'est en somme un électroscope dont la confection est facile et dont le prix de revient ne dépasse pas 50 centimes.

L'électroscope peut être tenu à la main sans danger, car il n'a pas besoin d'être amené au contact des conducteurs chargés, sauf s'ils le sont à basse tension. - En approchant la boule de cuivre à 2 ou $3 \mathrm{~cm}$ d'un conducteur chargé à 2500 volts, la répulsion des feuilles d'aluminium était presque totale.

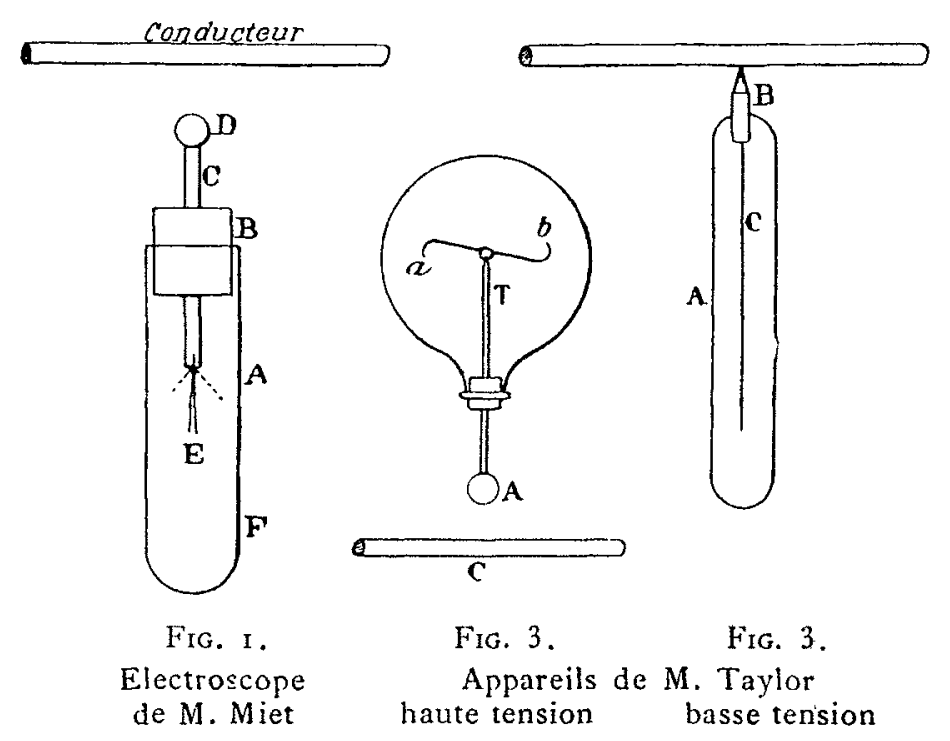

Lorsque le conducteur était porté à 10 ooo volts, la séparation des feuilles d'aluminium était très notable en tenant la boule de cuivre à $10 \mathrm{~cm}$ du conducteur.

D'ailleurs, si on mettait la boule en contact avec un conducteur, il ne se produirait pas d'inconvénient pour l'opérateur 
Le même appareil donnait une déviation déjà très sensible en le mettant au contact d'un conducteur nu chargé au potentiel de roo volts continu, l'autre pôle de la canalisation étant relié au sol.

En résumé, l'appareil Miet est très sensible et fonctionne entre des limites de tension très étendues de 100 à 20000 volts.

Pour de très hautes tensions, l'inventeur indique qu'on peut dévisser la boule de cuivre et la remplacer par un bloc de bois dur. La sensibilité reste suffisante pour les hautes tensions et le danger est nul pour l'opérateur. Cet appareil répond donc bien aux conditions du programme.

Les expériences qui précèdent ont porté sur l'essai d'un conducteur unique, isolé ou non, armé ou non. Il va de soi que les appareils du Concours ne peuvent fournir aucune, indication axec les câbles armés concentriques, puisque ces derniers ne donnent lieu à aucun champ extérieur.

Appareils de M. Taylor. - Les appareils de M. Taylor se divisent en appareils pour haute tension et en appareils pour basse tension.

$1^{\circ}$ L'appareil pour haute tension (fig. 2) est constitué par le classique tourniquet électrique fondé sur l'écoulement de l'électricité par les pointes. Il se compose d'un S en fil métallique léger formant le tourniquet proprement dit; l'S est montée sur une chape reposant sur une tige métallique $\mathrm{T}$.

Les extrémités $a$ et $b$ du tourniquet sont effilées en pointe aiguë.

L'ensemble est abrité par un ballon de verre de 7 à $8 \mathrm{~cm}$ de diamètre. Si l'on met la partie inférieure $A$ de la tige $T$ en contact avec un conducteur $\mathrm{C}$ sous tension, le tourniquet se met à tourner. - Le contact avec le conducteur de haute tension est nécessaire, et par conséquent, l'appareil est inefficace en présence d'un conducteur isolé.

Le tourniquet électrique soumis aux essais ne commencait à tourner que lorsque la tension atteignait 7000 volts. $\dot{A}$ des tensions plus élevées, la vitesse du tourniquet augmentait sensiblement.

Dans sa notice descriptive, $M$. Taylor indique qu'il a réussi à construire un tourniquet fonctionnant à partir de I 500 volts.

L'appareil exigeant d'être mis en contact avec les conducteurs chargés, il est imprudent de s'en servir pour les lignes aériennes. Il ne donne pas de résultats avec les câbles isolés,

Son emploi paraît tout indiqué à poste fixe pour les tableaux de distribution ou les stations de transformateurs.

L'instrument ne répond donc qu'à une partie du programme.

$2^{\circ}$ Appareil pour courants à basse tension. - Ces appareils (fig. 3) se composent d'un tube de verre A de 7 à $8 \mathrm{~cm}$. de long et de $1 \mathrm{~cm}$ de diamètre, fermé à un bout. Le tube est rempli de gazoline tenant en suspension de l'or massif (bisulfure d'étain) ou des poudres d'aluminium ou de bronze. On ferme le tube avec un bouchon $B$, laissant passer une tige metallique $\mathrm{C}$ pénétrant dans le liquide.

Si on met la tige $C$ en contact avec un conducteur chargé à quelques centaines de volts, 'le rassemblement de la poudre est pour ainsi dire instantané.

On a remarqué que la sensibilité des tubes, c'est-à-dire la rapidité avec laquelle les poudres de bronze se réunissent, dépend de la fluidité du liquide et de la légèreté des poudres.
Les indications sont moins nettes avec le courant con tinu qu'avec l'alternatif'; pour les basses tensions, il fallait prêter beaucoup d'attention lorsqu'on ne dépassait pas 200 volts continu.

Le fonctionnement se produisait en approchant les tubes à quelques centimètres des conducteurs chargés à 6000 et 8000 volts.

En résumé, les résultats sont intéressants, mais les indications sont moins visibles qu'avec l'electroscope Miet. Si l'inventeur arrivait à rendre les phénomènes plus frappants, son indicateur pourrait rendre des services analo. gues à ceux des électroscopes.

Appareil de M. Thornton. - Cet appareil (fig. 4) consiste en un tube de verre T terminé par deux électrodes, comme un tube d'indicateur de pôles. Le liquide est cons. titué par de l'huile de pétrole tenant en suspension des filaments de drap carbonisé.

Lorsqu'on met les électrodes $\mathrm{AB}$ en contact avec les deux conducteurs d'une canalisation électrique, il passe un faible courant dans le tube $T$, les filaments de drap carbonisé s'orientent en forme de chaîne et de vives étincelles éclatent entre les fragments de drap. C'est une sorte de carreau étin. celant. Une poignée isolante sert à tenir le tube de manière à éviter tout danger pour l'opérateur.

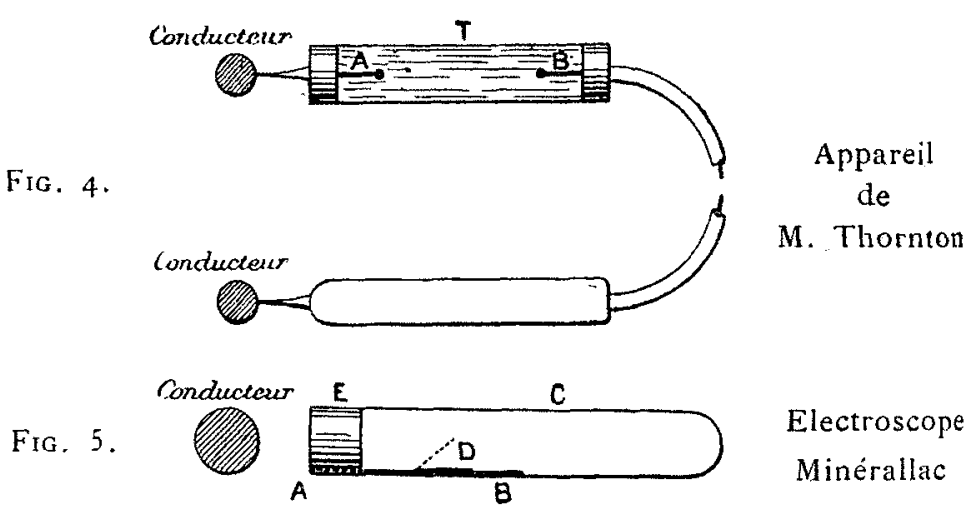

Cet appareil donne des étincelles visibles même par le soleil. Grâce à son manche isolant, on peut le manier sans danger, mais il présente l'inconvénient de nécessiter la mise en contact avec les deux conducteurs nus d'une canalisation.

Appareil de la Minerallac $C^{0}$. - C'est un électroscope (fig. 5) analogue à celui de $M$. Miet; ses dimensions sont assez réduites pour qu'on puisse le porter dans une poche de gilet. Il se compose d'un tube de verre $C$ dont le bouchon E laisse pénétrer une lame de cuivre AC. Sur celle-ci repose une mince feuille d'aluminium $D$ normalement en contact avec la feuille de cuivre.

Si on approche la partie A d'un conducteur chargć, la feuille d'aluminium se soulève comme le montre la figure. Cet appareil fonctionne si les conducteurs sont sous tension (continue ou alternative). Il manque de sensibilité et ne paraît pas présenter une protection suffisante pour l'opérateur qui s'en sert. Cela tient surtout aux dimensions très réduites du tube de verre.

Néanmoins, cet appareil est d'un usage assez courant à Chicago, dans les usines Edison à haute tension. 